\title{
Stereolithographic model-assisted reconstruction of the mandibular condyle with a vascularized fibular flap following hemimandibulectomy: Evaluation of morphological and functional outcomes
}

\author{
HITOSHI YOSHIMURA ${ }^{1}$, SHINPEI MATSUDA ${ }^{1}$, SEIGO OHBA ${ }^{2}$, YOSHIKI MINEGISHI ${ }^{3}$, \\ KUNIHIRO NAKAI ${ }^{3}$, SHIGEHARU FUJIEDA ${ }^{4}$ and KAZUO SANO ${ }^{1}$
}

\author{
${ }^{1}$ Department of Dentistry and Oral Surgery, Unit of Sensory and Locomotor Medicine, Division of Medicine, \\ Faculty of Medical Sciences, University of Fukui, Fukui; ${ }^{2}$ Department of Regenerative Oral Surgery, \\ Graduate School of Biomedical Sciences, Nagasaki University, Nagasaki; ${ }^{3}$ Department of Plastic and \\ Reconstructive Surgery, University of Fukui Hospital, Fukui; ${ }^{4}$ Department of Otorhinolaryngology Head \\ and Neck Surgery, Unit of Sensory and Locomotor Medicine, Division of Medicine, \\ Faculty of Medical Sciences, University of Fukui, Fukui, Japan
}

Received May 13, 2017; Accepted August 25, 2017

DOI: 10.3892/ol.2017.6909

\begin{abstract}
The vascularized fibular flap is one of the standard treatment choices for the reconstruction of the mandible; however, the consequences of condylar restoration have not previously been reported. The use of three-dimensional models allows for a more predictable reconstruction. The purpose of the present study was to assess the outcome of stereolithographic model-assisted reconstruction of the mandibular condyle with a vascularized fibular flap. A total of 5 patients underwent mandibular resection including the condyle and immediate reconstruction with a vascularized fibular flap. A stereolithographic model was used to determine the length and angle of the bony reconstruction. In all patients, the temporomandibular joint (TMJ) disc was preserved, and the contoured fibular end was placed directly into the glenoid fossa under the TMJ disc. To investigate the morphological and functional outcomes, radiographic and clinical examinations were performed, and a food scale questionnaire was administered. The mean period of follow-up was 23 months, and all the flaps were viable. Cosmetic results were generally satisfactory. Radiographic assessment revealed that the end of the fibular graft became round-shaped. None of the patients had abnormal
\end{abstract}

Correspondence to: Dr Hitoshi Yoshimura, Department of Dentistry and Oral Surgery, Unit of Sensory and Locomotor Medicine, Division of Medicine, Faculty of Medical Sciences, University of Fukui, 23-3 Matsuoka, Shimoaizuki, Eiheiji-cho, Yoshida-gun, Fukui 910-1193, Japan

E-mail:omfs@u-fukui.ac.jp

Key words: stereolithographic model, mandible, condyle, temporomandibular joint, reconstruction, fibular flap bone resorption, dislocation or ankylosis. The mean value of maximum mouth opening was $31 \mathrm{~mm}$. No patients exhibited difficulties with occlusion. All patients recovered their ability to ingest nearly the same foods that were ingested prior to surgery. The stereolithographic model-assisted reconstruction of mandibular condyle with a vascularized fibular flap is therefore useful for morphological and functional reconstructions of the hemimandible, including condylar defects.

\section{Introduction}

The temporomandibular joint (TMJ) is the movable joint that connects the skull and mandible, and plays an important role in occlusion and mastication. TMJ is composed by the mandibular condyle fitting into the glenoid fossa of the temporal bone with the lining of the articular disc. This disc attaches to the medial and lateral poles of the condyle and facilitates the smooth movements of the mandible. During mouth opening, the condyle rotates underneath the disc, whereas the condyle-articular disc complex moves in a sliding movement relative to the temporal bone to articular eminence. Thus, the TMJ serves as a ginglymoarthrodial joint, and allows both wide vertical opening movement as well as lateral excursive movement of the mandible (1).

In the surgery of a mandibular tumor, resection with TMJ disarticulation and condylar removal are occasionally required to achieve an adequate surgical margin. However, because of the complex anatomic and functional features of the TMJ, the ideal restoration of the condyle is still subject to controversy, and reconstruction is a challenging issue for surgeons. The use of the vascularized fibular flap is one of the standard treatment choices for the reconstruction of the mandible. The method of condylar reconstruction with direct placement of a vascularized fibular flap into the glenoid fossa has been reported (2-13); however, few data have been accumulated 
about the consequence of TMJ restoration. In some reported cases, the unsuitable shape or location of the graft disturbed TMJ function (7), and then affected the patients' quality of life.

Recently, computer-aided technology has become available and has given the surgeon the ability to plan the bony reconstruction and to prepare intraoperative guidance preoperatively, which can assist the surgeon (14). Although mandibular reconstruction remains a challenging aspect that depends on the surgeon's experience, intraoperative judgment, and technical speed, the stereolithographic model can reduce learning curve associated with neomandibular countering, enhance the levels of accuracy, and accelerate a time-consuming operative step, which then allows a more predictable reconstruction (15).

In this study, we report a stereolithographic model-assisted reconstruction of the mandibular condyle with direct placement of a vascularized free fibula into the glenoid fossa. The stereolithographic model was used for an anatomical template for mandibular condylar reconstruction. To investigate the postoperative the morphological and functional outcomes of the TMJ, clinical and radiographic examinations were performed. We also administered a food scale questionnaire (16) to evaluate the masticatory function. Furthermore, we reviewed the previous reports of condylar reconstruction with a fibular flap and discussed the ideal restoration of the condyle in the cases where the condyles are resected.

\section{Materials and methods}

Five patients underwent mandibular resection including the condyle and immediate reconstruction with a vascularized fibular flap at our hospital from September 2013 to July 2015 (Table I). There were two men and three women whose ages ranged from 44 to 74 years (mean age, 61 years). The lesions of five cases were located in the posterior mandible; four cases were squamous cell carcinomas, and one case was keratocystic odontogenic tumor.

The computed tomography (CT) scanner used in this study was SOMATOM Definition AS+ (Siemens Healthcare Diagnostics, Tokyo, Japan) or Discovery CT750 HD (GE Healthcare, Tokyo Japan). All patients underwent CT scanning (1-mm slice thickness) of the whole mandible and $5 \mathrm{~cm}$ above the glenoid fossa before surgery. The data from CT scanning in Digital Imaging and Communications in Medicine (DICOM) format were sent to a medical modeling company (AHEAD Laboratories, Tokyo, Japan), and the stereolithography model of the mandible was reconstructed. The resin template was also fabricated from the stereolithography model in our dental laboratory and sterilized for intraoperative use.

In all patients, a hemimandibulectomy was performed with margins to isolate the tumor. Four patients also underwent neck dissection. In all patients, the masseter and the internal pterygoid muscles were resected, and the TMJ disc was preserved. The defect patterns were classified into four cases of $\mathrm{CRBS}^{\mathrm{H}}$ and one case of CRB; the letters C, R, B, and S indicate defects of the condyle, ramus, body and symphyseal regions and the superscript ' $H$ ' indicate a hemisymphyseal defect (17). The mean bony defects was $129 \mathrm{~mm}$ (range, 93-141 mm).

All cases were reconstructed with fibular osteocutaneous flaps. A 3D resin model was used to determine the length of segments and the angle of bony reconstruction. A single osteotomy of the fibula was performed to recreate the mandibular shape. In all cases, the fibular end was placed into the glenoid fossa under the TMJ disc. The residual mandibular segment was temporarily fixed with maxillomandibular fixation (MMF), and the fibular graft was fixed to the residual mandible with titanium plates and screws. Then, the outer contour of the mandible (inferior-lateral mandibular border) was restored. In all patients, splints or dentures were utilized to keep occlusion during MMF. Microvascular anastomoses were completed using a microscope. The postoperative MMF was applied for 10-21 days (mean, 15.8 days). After the release of MMF, the patients were allowed to start masticating a soft diet, and they began active jaw mobilization with a rehabilitation device of mouth opening. Four cases received radiation therapy preoperatively and/or postoperatively. Panoramic radiograph examination was performed during the follow-up period.

The flap survival rates were examined and the postoperative complications were reviewed. The facial contour was evaluated by two of the authors. To investigate the postoperative morphological and functional outcomes of the TMJ, radiographic and clinical examinations were performed. Evaluation was performed at least one year after the initial reconstruction. In the radiographic evaluation, the morphologic changes of reconstructed condyle were traced using panoramic radiographs, and the presence of rounding, abnormal bone resorption, dislocation, and ankylosis were examined. To evaluate the abnormal bone resorption, the measurments were made between the same fixation point on the lower border of the flap and the medial point on the fibular end (7).

In the clinical evaluation, occlusion, maximum mouth opening (MMO), mouth opening pattern (midline deviation), and TMJ symptoms including pain, muscle pain with palpation (muscles of mastication and others), jaw joint noises (clicking or crepitation) and closed or open locking of the jaw of the patients were examined.

To evaluate masticatory performance, tooth condition was examined, and a food scale questionnaire (16) was performed (Table II). The number of natural teeth, and the prior use of prostheses were examined. Tooth-to-tooth contact was evaluated by occluding paper, and the pairs of occluding teeth were noted. The food scale questionnaire used in this study has demonstrated sufficient sensitivity for the evaluation of functional differences between head and neck cancer patients $(16,18)$.

The study was approved by the Institutional Research Board (Ethical Committee of the University of Fukui, Faculty of Medical Sciences; no. 20170056). The patients provided informed consent for the usage of the data in this study.

Surgical technique. The stereolithography model was made before the operation. The resin template was also fabricated from the stereolithography model in the dental laboratory (Fig. 1A). Intermaxillary fixation (IMF) screws (Dual-Top Auto Screw; Jeil Medical Corporation, Seoul, South Korea) were used during MMFs. MMF was once performed to recognize the maxillomandibular relationship and occlusion before the resection of the mandible. A splint or denture was applied in patients who were partially or full edentate. Next, the MMF was released, and a hemimandibulectomy was performed 


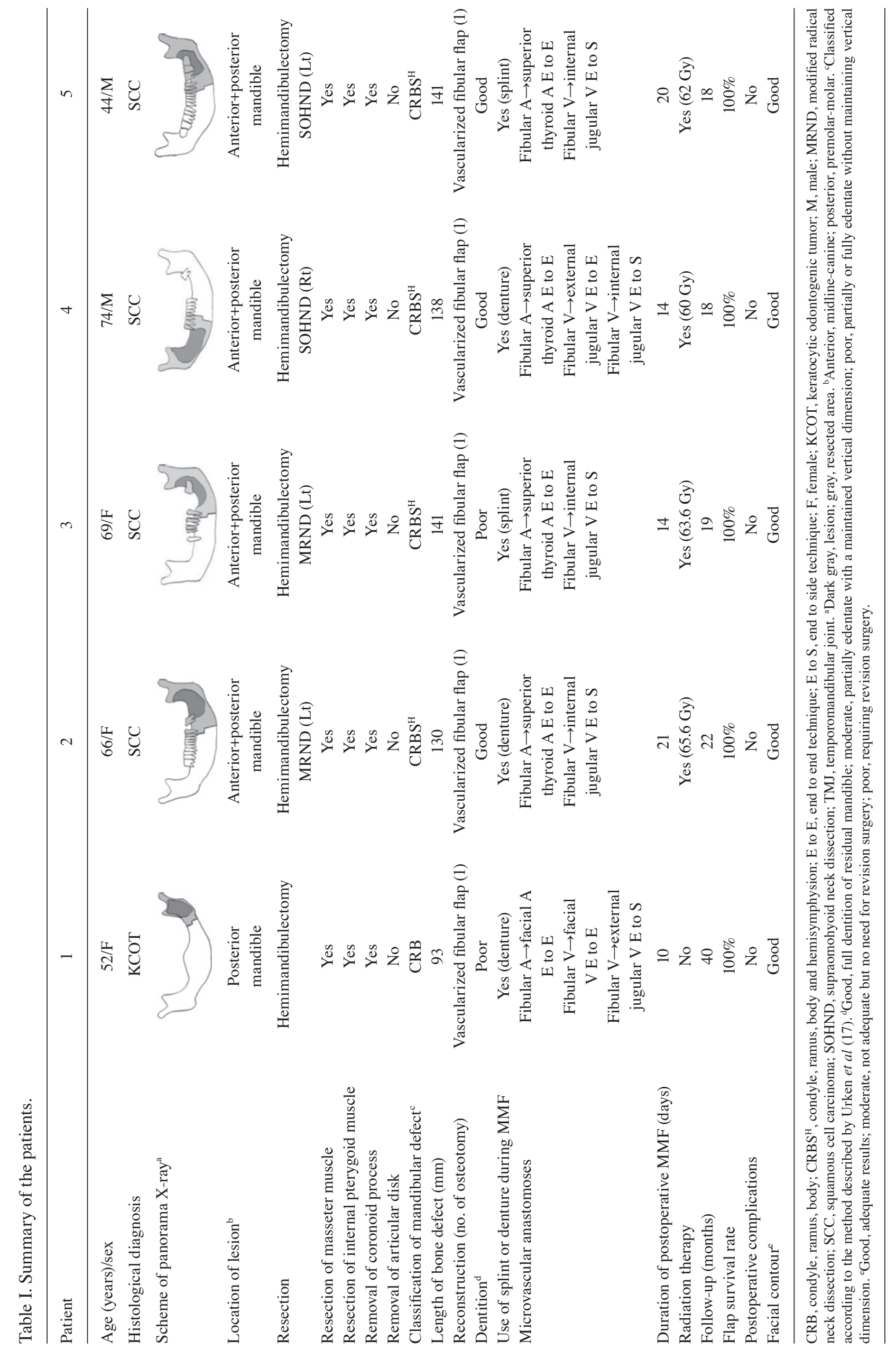



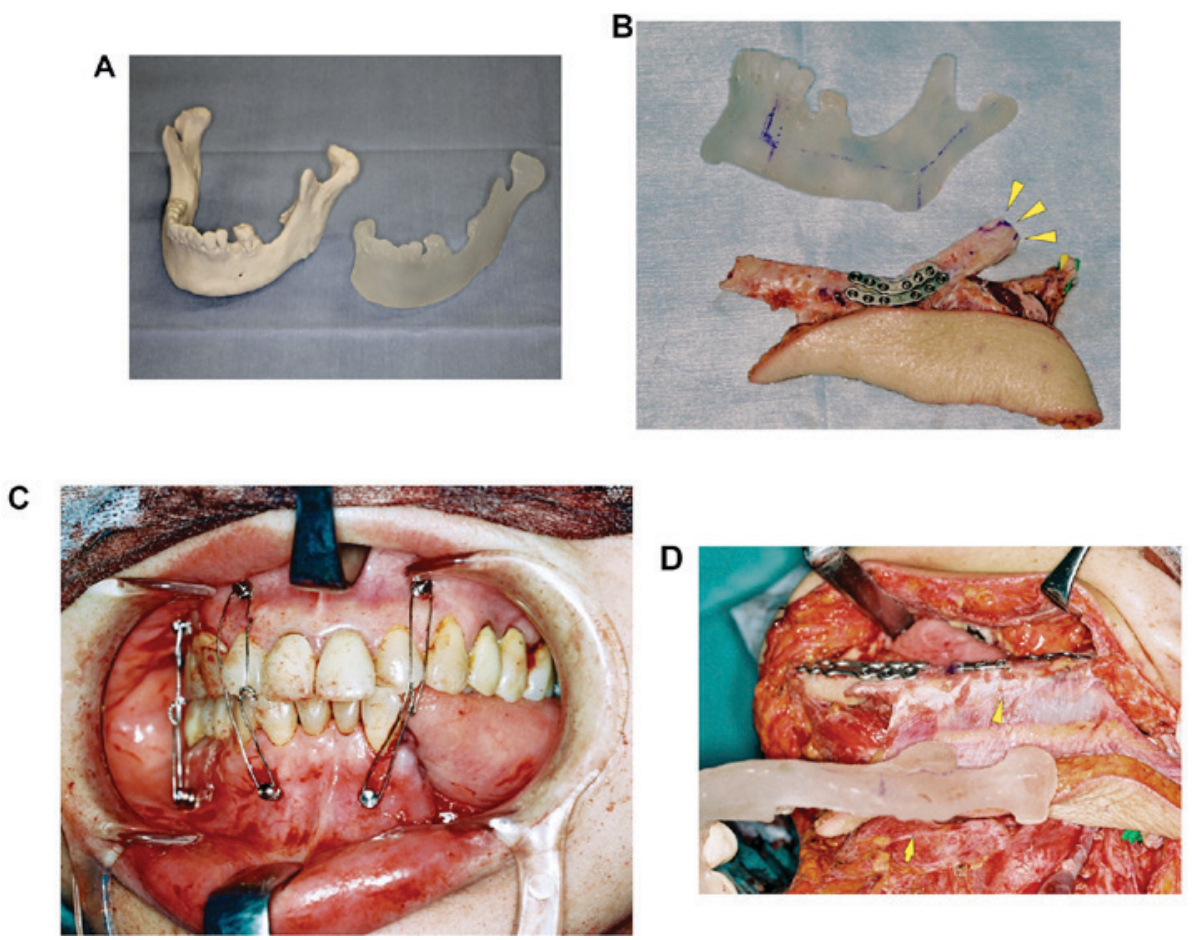

Figure 1. (A) The stereolithography model of the mandible was reconstructed. The resin template was also fabricated from the stereolithography model in dental laboratory. (B) A fibular osteocutaneous flap was harvested and adjusted to the defect. A 3D resin model was used to determine the length of the segments and the angle of the bony reconstruction. The straight fibula bone was osteotomized to match the form of the mandible. The fibular end was adjusted to a round shape to fit into the glenoid fossa (arrowheads). (C) Prior to reconstruction, the residual mandibular segment was held with MMF in a 3D fashion. Then, the condyle of non-affected side was seated in the appropreate position, and the correct occlusion was reestablished. The use of MMF allowed the fibula to be inserted under the temporomandibular disc in the glenoid fossa. (D) The fibular osteocutaneous flap and the mandible remnants were fixed with titanium miniplates and screws. The fibular end was placed into the glenoid fossa under the TMJ disc. A 3D resin model was used to adjust the position of fibular flap (arrowhead). MMF, maxillomandibular fixation; TMJ, temporomandibular joint.

to isolate the tumor. The temporalis and masseter muscles were resected; the medial and lateral pterygoid muscles were resected; and the remaining soft tissue surrounding the condyle was removed. In all cases, the temporomandibular disc was preserved. Then, a fibular osteocutaneous flap was harvested and adjusted to the defect. A 3D resin model was used to determine the length of segments and the angle of bony reconstruction. The straight fibula bone was osteotomied to match the form of the mandible. The fibular end was adjusted to a round shape to fit into the glenoid fossa (Fig. 1B). The residual mandibular segments were repositioned by MMF (Fig. 1C). The fibular end was placed into the glenoid fossa under the TMJ disc. During the operation, care was taken to place the residual mandible and the lower edge of the transplanted fibula at the same level. The distance from the angle of the mandible to the angle of the fibular flap was corrected to be the same length as the 3D model. The fibular graft was secured to the residual mandible with titanium plates and screws (Fig. 1D). A 1.0-1.5 mm thick straight or curved-shaped plate and 5 or $6 \mathrm{~mm}$ screws (MatrixMANDIBLE; DePuy Synthes Co., Tokyo, Japan) were used. After positioning the fibula, the lateral pterygoid muscle was not secured to the fibula with sutures. The masseter muscle was also not sutured to the internal pterygoid muscle to actively seat the flap into the glenoid fossa. Next, microvascular anastomoses were performed using a microscope. Then, the MMF was released, and mandibular movement, dental occlusion, and the position of the neocondyle were confirmed. Patients underwent
Table II. Food scale questionnaire ${ }^{\mathrm{a}}$.

\begin{tabular}{ll}
\hline Rating & Most difficult food patient is able to masticate \\
\hline 100 & Full diet (no restrictions) \\
90 & Peanuts \\
80 & All meat \\
70 & Carrots, celery \\
60 & Dry bread and crackers \\
50 & Soft, chewable foods \\
40 & Soft foods requiring no chewing \\
30 & Pureed foods (in blender) \\
20 & Warm liquids \\
10 & Cold liquids \\
0 & Nonoral feeding (tube fed)
\end{tabular}

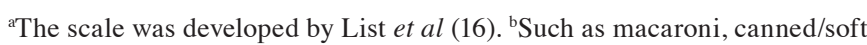
fruits, cooked vegetables, fish, hamburger, small pieces of meat. 'Such as mashed potatoes, apple sauce, pudding.

postoperative MMF for 10-21 days, followed by mobilization and jaw exercises.

\section{Results}

The mean follow-up duration was 23.4 months (range, 18-40 months) (Table I). In all cases, the tumor was completely removed with a clear margin. There was no instance of vascular 
Table III. Morphological and functional evaluation of the temporomandibular joint.

\begin{tabular}{|c|c|c|c|c|c|}
\hline Patient & 1 & 2 & 3 & 4 & 5 \\
\hline \multicolumn{6}{|l|}{ Morphological evaluation } \\
\hline Dislocation & No & No & No & No & No \\
\hline Ankylosis & No & No & No & No & No \\
\hline Change into round-shape of fibular end & Yes & Yes & Yes & Yes & Yes \\
\hline Abnormal bone resorption & No & No & No & No & No \\
\hline \multicolumn{6}{|l|}{ Functional evaluation } \\
\hline Occlusion & Centric & Centric & Centric & Centric & Centric \\
\hline MMO (early postoprative period, $\mathrm{mm})^{\mathrm{a}}$ & 23 & 13 & 20 & 15 & 18 \\
\hline MMO (late postoperative period, $\mathrm{mm})^{\mathrm{b}}$ & 40 & 21 & 25 & 38 & 32 \\
\hline Mouth opening pattern & $\begin{array}{l}\text { Minimal } \\
\text { deviation }\end{array}$ & $\begin{array}{l}\text { Minimal } \\
\text { deviation }\end{array}$ & $\begin{array}{l}\text { Minimal } \\
\text { deviation }\end{array}$ & $\begin{array}{l}\text { Minimal } \\
\text { deviation }\end{array}$ & $\begin{array}{l}\text { Minimal } \\
\text { deviation }\end{array}$ \\
\hline Lateral movement (affected side, $\mathrm{mm}$ ) & 8 & 1 & 2 & 4 & 5 \\
\hline Lateral movement (non-affected side, $\mathrm{mm}$ ) & 6 & 1 & 2 & 3 & 3 \\
\hline Protrusive movement, $\mathrm{mm}$ & 7 & 0 & 0 & 4 & 5 \\
\hline TMJ pain & No & No & No & No & No \\
\hline Muscle pain with palpation & No & No & No & No & No \\
\hline Jaw joint noise & No & No & No & No & No \\
\hline Closed or open locking of the jaw & No & No & No & No & No \\
\hline
\end{tabular}

MMO, maximal mouth opeining; TMJ, temporomandibular joint. ${ }^{a}$ At the time of MMF release; bat least 12 months of postoperation.

Table IV. Assessment of masticatory function.

\begin{tabular}{|c|c|c|c|c|c|c|c|}
\hline Status & Patient & 1 & 2 & 3 & 4 & 5 & Average \\
\hline \multirow[t]{5}{*}{ Preoperative status } & Number of maxillary natural teeth & 0 & 10 & 0 & 11 & 13 & 6.8 \\
\hline & Number of mandibular natural teeth & 0 & 11 & 7 & 9 & 15 & 8.4 \\
\hline & Use of prostheses (removable dentures) & No & Yes & Yes & Yes & No & $60 \%$ (usage rate) \\
\hline & Number of contacted teeth ${ }^{\mathrm{a}}$ & 0 & 13 & 6 & 14 & 13 & 9.2 \\
\hline & Food scale rating & 50 & 100 & 80 & 100 & 100 & 86 \\
\hline \multirow[t]{5}{*}{ Postoperative status } & Number of natural maxillary teeth & 0 & 10 & 0 & 9 & 13 & 6.4 \\
\hline & Number of natural mandibular teeth & 0 & 8 & 2 & 5 & 9 & 4.8 \\
\hline & Use of prostheses (removable dentures) & Yes & Yes & No & Yes & Yes & $80 \%$ (usage rate) \\
\hline & Number of contacted teeth ${ }^{\mathrm{a}}$ & 14 & 13 & 2 & 12 & 13 & 10.8 \\
\hline & Food scale rating & 100 & 90 & 70 & 80 & 100 & 88 \\
\hline
\end{tabular}

ancluding the teeth of prostheses (removable dentures).

compromise, and the flap survival rate was $100 \%$. There was no extrusion of hardware, breakage of the titanium plate, or screw looseness. All patients had a stable mandibular union and good to moderate facial contour. No patients experienced facial nerve paresis. All patients maintained intelligible speech.

A radiographic assessment showed that the neocondyle remained in the glenoid fossa, and there was no dislocation or ankylosis in any of the patients (Table III). Remodeling of the end of the neocondyle was found, and the shape was changed to resemble a round shape. Twelve months after the surgery, measurement of length between the fixed point on the lower border of the flap and the median point on the upper side of the neocondyle showed a decrease of less than $5 \%$ of the early postoperative measurements. Thus, there were no abnormal bone resorptions.
In all patients, the mandible was placed in centric occlusion. The mean MMO at the time of MMF release was $17.8 \mathrm{~mm}$ (range, 13-23 mm), and at least 12 months postoperatively, it was $31.2 \mathrm{~mm}$ (range, 21-40 $\mathrm{mm}$ ). All patients showed an improvement of MMO of more than $5 \mathrm{~mm}$ during the follow-up period. All the patients had a slight lateral shift from the midline to the affected side during mouth opening. None of the patients had any TMJ pain, muscle pain with palpation, jaw joint noise, or closed and open locking of the jaw.

Masticatory function is presented in Table IV. Before surgery, the average numbers of natural maxillary and mandibular teeth were 6.8 (range, 0-13) and 8.4 (range, 0-15). After surgery, the average numbers of natural maxillary and mandibular teeth were 6.4 (range, 0-13) and 4.8 (range, 0-9). As a result of tumor resection, an average of 3.6 natural 
A

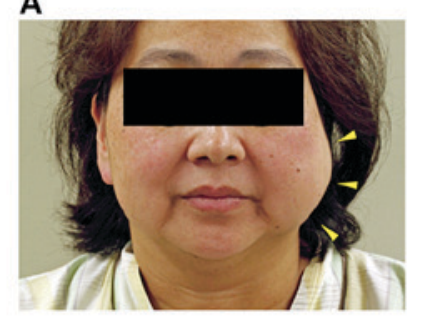

F

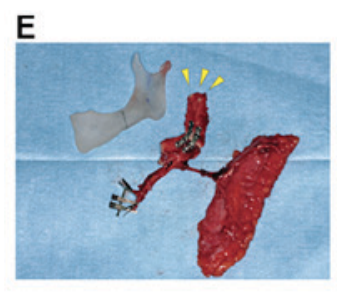

I

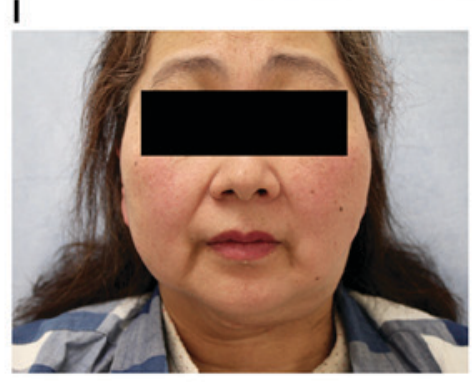

B

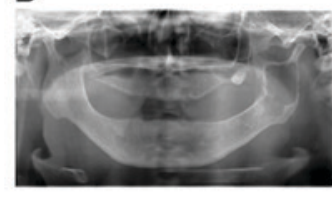

C

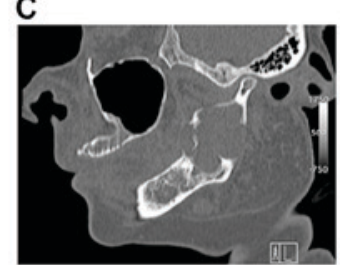

G

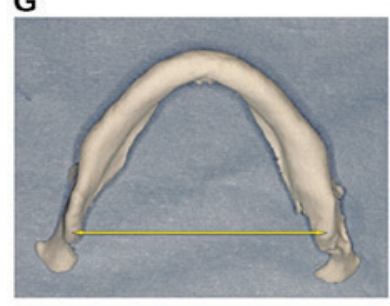

D

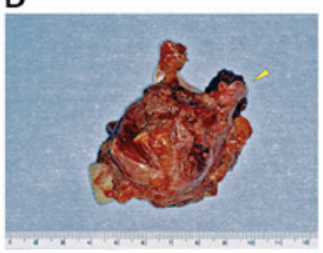

H

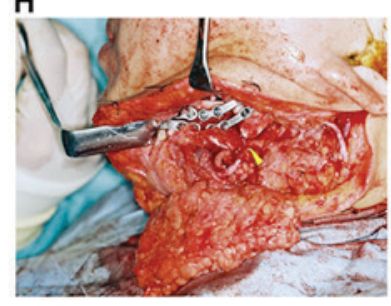

J

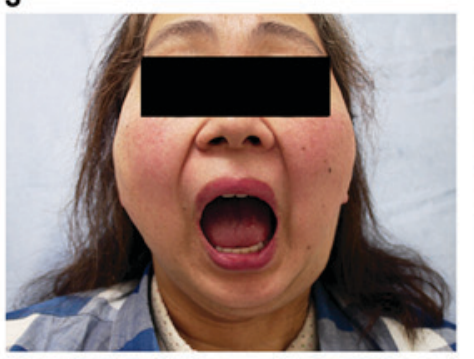

K

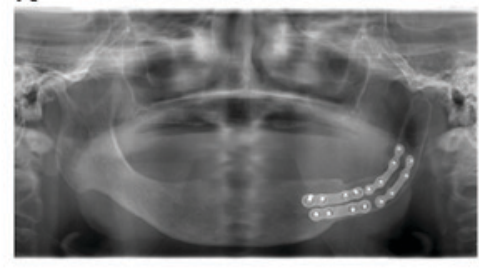

Figure 2. (A) Frontal view showing the swelling of the left mandible (arrowheads). (B) Preoperative panoramic radiograph showing the radiolucency throughout the mandibular ramus with extension near the condyle. (C) Preoperative CT showing a multilocular cystic lesion occupying the mandibular ramus and loss of cortical bone. (D) The resection was performed with the soft tissue covered over the lesion. Surgical specimen showing the deformity of the mandibular ramus due to the expansion. The condyle was disarticulated from the TMJ (arrowhead). (E) The fibular osteocutaneous flap was harvested and osteotomy was accomplished to shape the flap as a potential ramus and condyle in accordance with the 3-D resin model. The distal fibular end was rounded to allow it to fit the temporomandibular disc in the glenoid fossa (arrowheads). (F) The residual mandibular segment was repositioned by MMF with denture. (G) The width of the mandibular angle was measured in the 3-D model. $(\mathrm{H})$ The grafted bone was fixed with titanium miniplates. We paid attention to place the lower edge of the transplanted bone and mandible at the same level and to place the reconstructed condyle in the glenoid fossa with aid of the 3-D model. The distance from the angle of the mandible to the angle of the fibular flap was adjusted to be the same length of the 3-D model (arrowhead). (I) Findings after the reconstruction. Frontal view showing a good aesthetic result. $(\mathrm{J})$ The patient had excellent joint mobility and the MMO of denture teeth was 40 mm. The patient obtained sufficient mobility of the condyle. (K) The postoperative panoramic radiograph showed the optimal position of grafted bone in the glenoid fossa. There was no evidence of tumor recurrence. CT, computed tomography; MMF, maxillomandibular fixation; MMO, maximum mouth opening; TMJ, temporomandibular joint.

mandibular teeth (range, 0-6) were lost. Four of the five patients $(80 \%)$ used dentures as prostheses, and no patients were underwent the treatment of dental implants. The average number of occlusal teeth (including denture) was 9.2 preoperatively and 10.8 postoperatively. The mean food scale rating was 86 (range, 50-100) preoperatively, and 88 (range, 70-100) postoperatively. Therefore, all patients recovered their ability to ingest nearly the same foods that could be ingested before surgery.

Case report (case no. 1). A 52-year-old female visited our hospital due to the expansion and pain in the left mandible (Fig. 2A). Physical examination revealed no paresthesia in the left mental region. Oral examination showed a swelling of the left retromolar region without mucosal alteration. A panoramic radiograph revealed the radiolucency throughout the mandibular ramus with extension near the condyle (Fig. 2B). CT scanning showed a multilocular cystic lesion occupying the mandibular ramus and a loss of cortical bone (Fig. 2C). A biopsy confirmed the diagnosis of keratocystic odontogenic tumor (KCOT).
Considering the progression of the lesion, a mandibular resection, including condyle and reconstruction, was planned under general anesthesia. Before the mandibular resection, MMF with denture was carried out to evaluate the maxillomandibular relationship and released. The resection was performed with the soft tissue that covered the lesion. The condyle was disarticulated and the articular disc was preserved (Fig. 2D). The fibular osteocutaneous flap was harvested, and osteotomy was accomplished to shape the flap as a substitute ramus and condyle in accordance with the 3D resin model (Fig. 2E). The distal fibular end was rounded to allow it to fit the temporomandibular disc in the glenoid fossa. The residual mandibular segment was repositioned by MMF with a denture (Fig. 2F). The width of the mandibular angle was measured in the 3D model (Fig. 2G), and the distance from the angle of the mandible to the angle of the fibular flap was adjusted to be the same length (Fig. 2H). Then, the fibular osteocutaneous flap was fixed with titanium plates and screws.

The microvascular anastomosis was completed using a surgical microscope, and the wound was closed. MMF was 


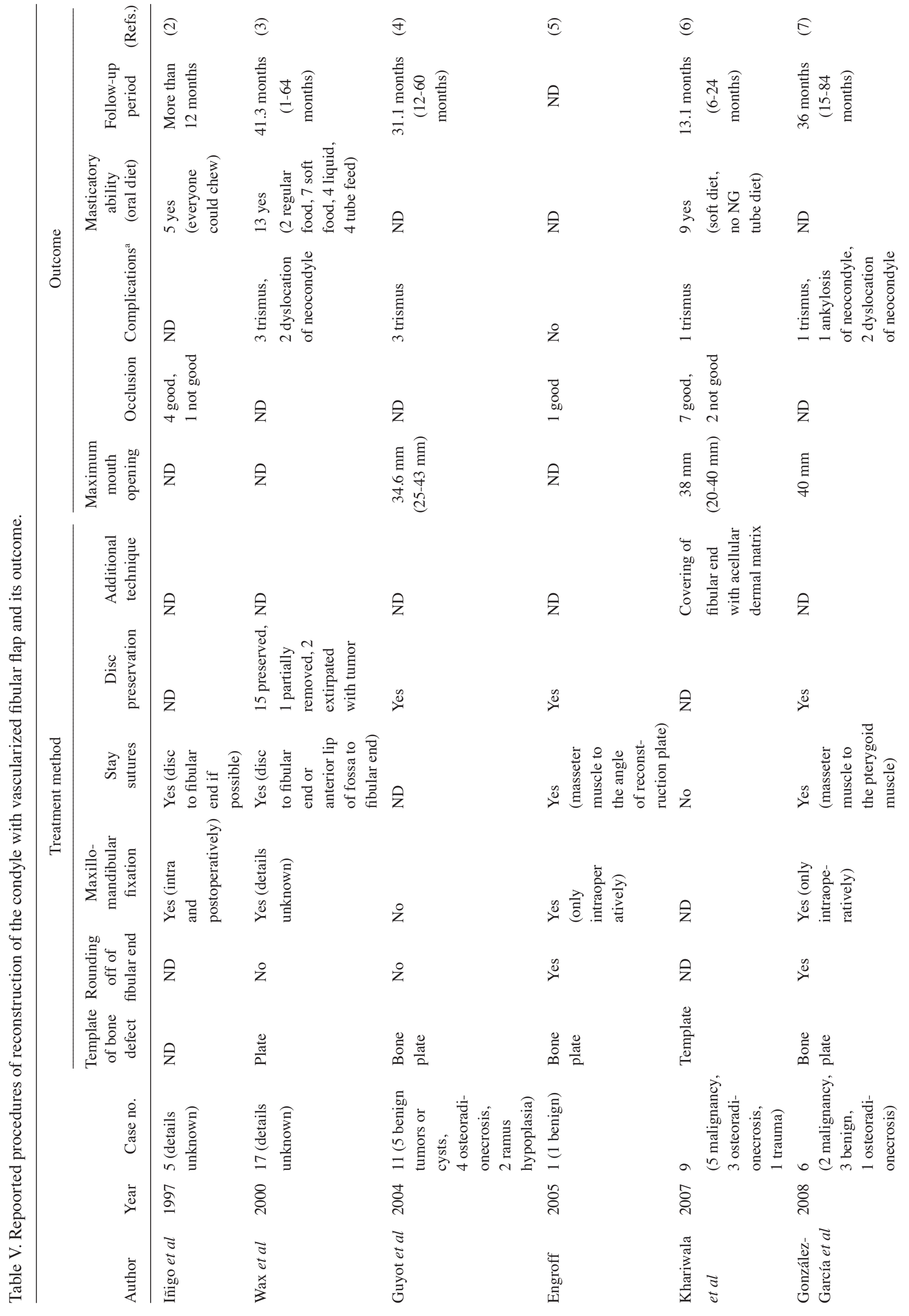




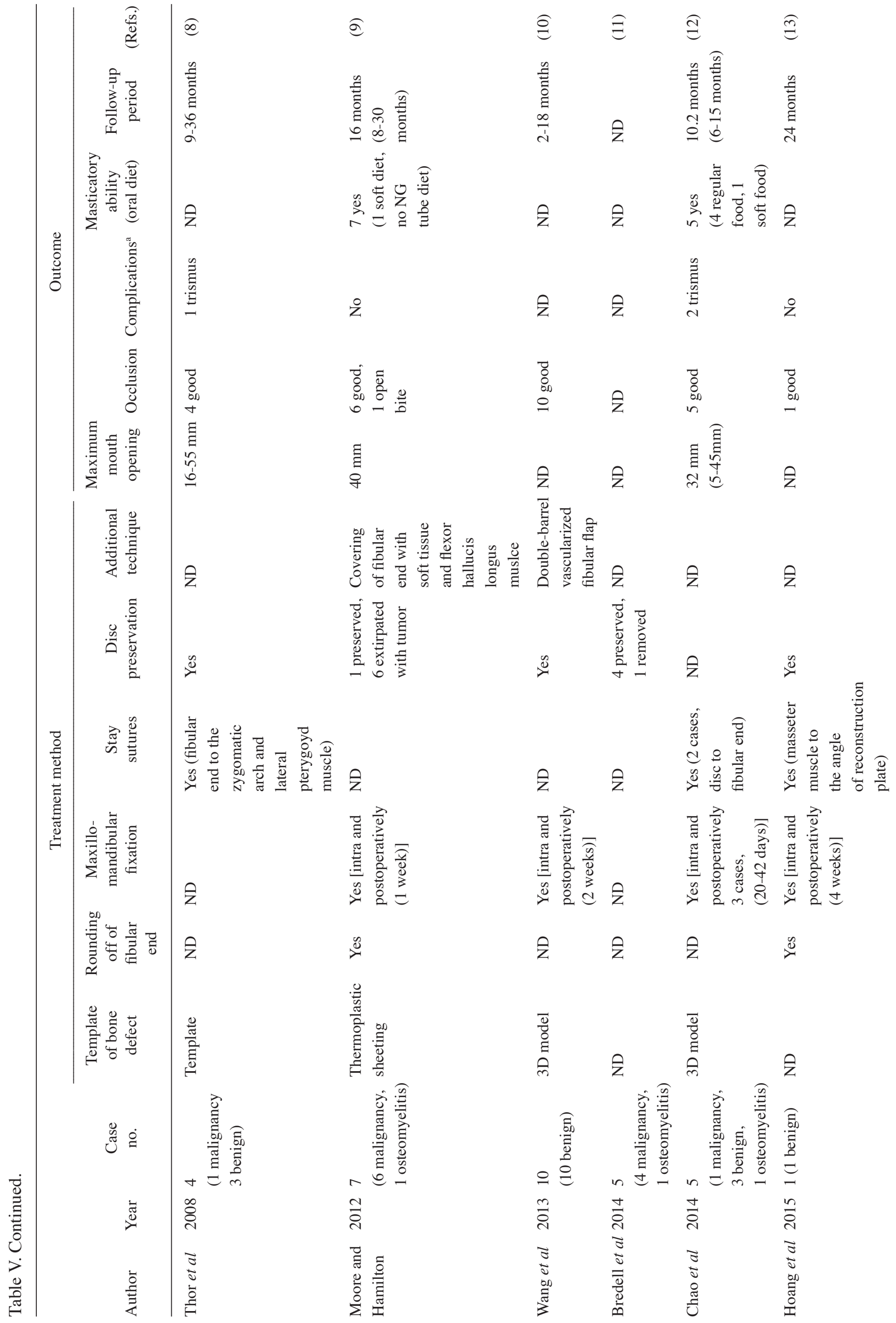


finally released 10 days after the operation, and the patient was discharged on 37 th postoperative day.

Forty months after the mandibular reconstruction, the patient showed good aesthetic results (Fig. 2I). She had excellent joint mobility and the MMO was $40 \mathrm{~mm}$ (Fig. 2J). The postoperative panoramic radiograph confirmed the optimal position of the grafted bone in the glenoid fossa, and showed the remodeled fibular segment in the condylar fossa (Fig. 2K).

\section{Discussion}

The condyle articulates with the glenoid fossa of the temporal bone and plays an important role in the proper function of the TMJ. The presence of an extensive tumor in the mandibular ramus or direct invasion of the condyle may require resection of the mandible with disarticulation of the TMJ. Because of the unique and complex features of the TMJ, the reconstruction of the condylar defect is one of the most challenging issues.

At present, several procedures are used for the reconstruction of the condyle, including costochondral graft, attachment of the resected condyle to the end of a graft, titanium TMJ prosthesis, and placement of the distal end of the vascularized graft directly into the glenoid fossa (9). Costochondral graft is one of the best methods for the transplantation of bone and choral components (19), but it is not large enough to replace bone after a complete hemimandibulectomy. Replacement with the resected condyle provides an excellent surface and fit for the joint, but concern exists regarding the oncologic risk, the condylar preservation during hemimandibulectomy may predispose the patient to local recurrence even with negative margins (20). Furthermore, these two types of grafts are devascularized, and the drawback is the possibility of abnormal resorption (5). The concern with the prosthetic condyle is the exposure of the prosthesis, risk of infection and erosion of the prosthesis into the middle cranial fossa $(21,22)$. Recently, the fibular graft has become the preferred procedure for mandibular reconstruction, because it is simple and can be easily fitted. Its use for the reconstruction of the condyle has been reported (Table V) (2-13). It seems particularly well suited for condylar reconstruction, because of its tubular shape and adaptability to the glenoid fossa. However, in some cases, complications were noted including poor mouth opening, mandible deviation, dislocation, abnormal bone resorption, ankylosis, and the need for additional surgical treatment (7). Thus, consensus has yet to be reached regarding the best method for reconstruction of the condyle with a fibular flap. In addition, little is reported about the outcome of the fibular flap in reconstructing the mandibular condyle.

Because of the unique feature of the TMJ, which is related to both the dentition and the contralateral TMJ, reproducing the optimal relationship between both the condyles (including neocondyle) and the glenoid fossa, and maintaining the correct occlusion are critical. Some authors reported the technique of using a bone plate for a template of the bone defect for the reconstruction of the mandibular condyle (3-5,7). However, this technique uses the outer surface of the mandibular bone as a template, and thus, it cannot be used when the lesion involves the outer cortex of the mandible. The use of a stereolithography model based on $\mathrm{CT}$ data has been also reported $(10,12)$. 

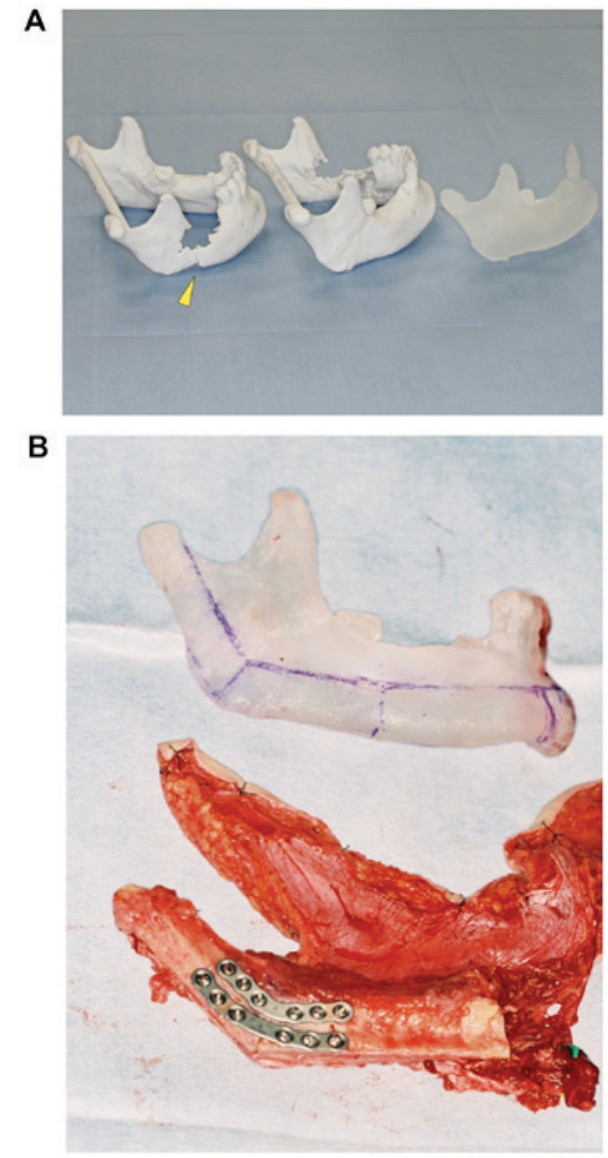

Figure 3. (A) The patient (case no. 4) showed the pathological fracture of the mandible due to the tumor progression. The stereolithography model of the mandible (left, arrowhead: fracture line), the mirror image of the stereolithography model (center), and 3D resin model of mirror-image (right). (B) A $3 \mathrm{D}$ resin model of the mirror-image was used to determine the length of segments and the angle of the bony reconstruction.

In this method, fabrication of surgical template can also be prepared by the mirror-image model of the unaffected side (Fig. 3A and B). This method is useful when intraoperative adaptation of the plate is impossible due to the size or position of the tumor. Using a 3D model, preoperative measurements can be made to determine the bone osteotomy for pathological resection, as well as to know the appropriate dimensions for bone reconstruction. By recognizing the defect dimensions before surgery, flap design and reliability, operative safety, and planning of the osteotomies can be improved. Furthermore, there is the possibility of reducing intraoperative decision-making, and thus potentially contributing to a significant shortening of the operative time, which is associated with complications (23). Thus, a 3D model can improve patient outcomes with a reduction in complications.

Nahabedian et al reported that the technical limitations of condylar reconstruction with a fibular flap made it difficult to reproduce the 3D shape of the native condyle (24). However, in our cases, contouring the fibular pole was accomplished to shape the flap as a substitute condyle in accordance with the $3 \mathrm{D}$ resin model. It may also facilitate the appropriate fit in the glenoid fossa and make a functional condyle. Although the cost of a 3D model is not negligible (at the time of the preparation of this article, the costs of creating a 3D model began at $¥ 20,000$ ), it is decreasing and becoming more readily available.

Currently, some surgeons prefer to use cutting guides to perform the osteotomy and fibula shaping. This method is faster and more reliable surgical results (25). However, one of the concerns of the cutting guide is the unpredictability of the surgical margins in some cases (26). The changing the extent of resection may reduce the usefulness of this method by the need of adjustments in bone segment length, number of osteotomies, and the shape of the titanium plate, potentially increasing the operation time and decreasing the reconstructive accuracy (26). In addition, since the device is designed according to the location of the fibular bone, it may be difficult to use a cutting guide if soft tissue is included with the fibula for oral or facial reconstructions.

Lim et al reported that the maximum error was observed in the condylar region when only a fibula-cutting guide was used (27). This error was thought to be related with the movement of the condylar head in the TMJ (27). They suggested that it may be more helpful to adjust the anterior-posterior position of the fibular bone by referring to the $3 \mathrm{D}$ model rather than using a cutting guide (27).

During the reconstruction of mandible, the optimal correlation of the condyles in the glenoid fossae and accurate occlusion are important. When a defect of hemimandible occurs, the masticatory muscles attached to the remaining segment will shift the segment into an unusual position. Therefore, the residual mandible should be relocated during the reconstruction. Intraoperative MMF is helpful for maintaining the occlusal and maxillomandibular relationship during reconstruction (28). In the case of edentulous or partially edentulous patients, dentures or splints are useful for reinforcing the remaining teeth and maintaining the dimensions of the maxillomandibular relationship $(29,30)$. In this study, five patients were edentate, or poor dentition, and dentures or splints were used to restore the occlusal relationship during MMF.

It is also a surgical challenge to maintain the fibular end in the glenoid fossa during and after reconstruction. In our cases, the use of MMF allows direct appliance of the fibula to the temporomandibular disc in the glenoid fossa. The distance from the angle of the mandible to the angle of the fibular flap was adjusted to be the same length as the $3 \mathrm{D}$ model. Then, the fibular graft was fixed to the residual mandible with titanium plates and screws. In this method, smooth insetting, anastomosis, and the planning of flap design, can be performed, and it may decrease the complication rate. In our cases, no instances of vascular compromise or fistula formation was observed. The 3D model could accurately and safely reproduce the 3D shape and the anatomical location of the original condyle with the aid of MMF.

Some investigators have reported the suture fixation, such as the suture of the fibular end to the preserved disc $(2,3)$, the suture of the fibula or the reconstruction plate to the masseter or pterygoid muscles $(5,8,13)$, and the suture of the masseter muscle to the pterygoid muscle (7). However, suspension of the fibular flap in the fossa with sutures is sometimes difficult and inaccurate (5). In addition, the remaining masseter or pterygoid muscles is occasionally not enough for suture due to the tumor resection. The displacement of the fibular flap has been reported in cases with suture fixation (7). In our cases, we 
chose to perform MMF for 2-3 weeks followed by mobilization in a manner similar to rehabilitation. In this method, MMF can maintain occlusal status and the maxillomandibular relationship, and hold the reconstructed mandible during the healing process. None of our patients experienced displacement of the fibula out of the condylar fossa. Chao et al also reported the use of postoperative MMF to condylar reconstruction with a fibular flap for 20-42 days and showed good results (12). These results indicate that MMF may contribute to the functionality of reconstruction through the recovery of accurate biting.

The temporomandibular disc is a biconcave sheet of avascular fibrous connective tissue. The disc divides the joint into a superior and inferior joint space and prevents direct contact of the condylar head to the mandibular fossa. The previous study showed that the preservation of the temporomandibular disc influences the postoperative recovery of normal function (12). Furthermore, the temporomandibular disc is important to control the form of the mandibular condyle (31). Guyot et al reported on 11 patients undergoing reconstruction with a fibular flap following condylar resection without the removal of the disc. In all cases, the fibular end was insetted directly into the glenoid fossa under the disc without contouring (4). In their cases, TMJ function was preserved, and there were no cases of ankylosis. They also found the tendency of bone remodeling. In our cases, the temporomandibular disc was preserved in all patients, and the round-off of the fibular ends without ankylosis were found. The most likely reason for these changes is considered to be the preservation of TMJ disc.

In a previous study, González-García et al (7) showed six patients who underwent the mandible and condyle resection without disc resection and reconstruction with fibular flap. They found that five patients achieved good postoperative function, but one patient experienced severe ankylosis. The temporomandibular disc blends with fibers of the lateral pterygoid muscle at its anterior margin, and attaches to looser connective tissue containing nerves and is lined posteriorly with the synovial membrane. Hamada et al performed the magnetic resonance imaging (MRI) and arthroscopy in the TMJ of two patients who experienced hemimandibulectomy without disc removal (32). The MRI of the affected TMJs showed that the intermediate zone of the disc was positioned anteriorly to the summit of the articular eminence. Arthroscopically, the fibrous adhesions were also observed in the superior joint compartment of the affected TMJs. They suggested that the disc was pulled anteriorly by the lateral pterygoid muscle, and they also thought that surgical trauma during mandibulectomy brought synovitis or hematoma, subsequent immobilization of the affected TMJs, and aroused intraarticular fibrous adhesion. As shown in other previous reports, not only the disc removal, but also the disc displacement or articular damage was associated with the development of ankylosis (33-36). Thus, the possibility of ankylosis must be kept in mind even in the case of disc preservation. In cases where disc removal is necessary due to disease progression, covering the contoured end of the fibula with adequate vascularized soft tissue could overcome this problem (9). Moore and Hamilton reported that none of their six patients had ankylosis in this technique (9).

Generally, vascularized bone graft suffers less resorption than non-vascularized bone graft (5). We performed length measurements using panoramic radiographs and revealed that no abnormal resorption of the neocondyle occurred. Chao et al reported the usage of a resected condyle to obtain adequate TMJ function, but the native condyle became avascular necrosis and was removed (12). In our opinion, it would seem unnecessary to attach the native (original) condyle to the fibular flap.

To objectively evaluate treatment outcomes, we assessed the TMJ and dental condition, conducted a food scale questionnaire (12), and examined masticatory status. In our study, the MMO of three patients was greater than $30 \mathrm{~mm}$, but two patients exhibited postoperative trismus with less than $30 \mathrm{~mm}$ of MMO. Radiation therapy commonly influences a patient's mandibular movements (37). These two patients received radiotherapy and developed limitation of mandibular sliding mobility and mouth opening. It was considered to be an adverse effect of radiation therapy. The forward movement is caused by the contraction of the inferior head of the lateral pterygoid muscle. The condyle and disc then move forward until they reach a point just slightly anterior to the crest of the articular eminence (38). In all our patients, the lateral pterygoid muscle was partially resected and not sutured with fibular end; thus, forward movement was considered to be difficult to achieve. The mouth opening-closing path showed a tendency to deviate toward the affected side, which was considered to be due to movement restriction on the affected side. Early exercise therapy is essential in surgery involving the TMJ (5). Our patients underwent mobilization and rehabilitation exercises after the release of MMF, and MMO improved as jaw physiotherapy progressed. The position of the condylar axis is related to symptomatology of TMJ (39), and mandibular resection may cause TMJ disorder (40). In this method, both condylar positions were kept by MMF, and TMJ disorder was not observed.

Mastication comprises synchronous interaction between the orofacial soft and hard tissues to manipulate, triturate, and consolidate a food bolus prior to deglutition (37). The mandible plays an critical role in the competence of mastication (37). After resection of the mandible, masticatory function can be affected by several factors such as the number of residual teeth and tooth-to-tooth contacts $(37,41,42)$. To objectively assess our outcomes, masticatory function was evaluated with reference to dental status, and was examined using a food scale questionnaire. In our patients, an average of 3.6 natural mandibular teeth was lost due to the tumor resection. Four patients $(80 \%)$ were treated with removable dentures postoperatively, and tooth-to-tooth contact was achieved. The food scale questionnaire showed that the patients recovered their ability to ingest nearly the same foods that can be ingested before surgery. Tan et al (43) showed that postoperative restoration with removable dentures significantly improved masticatory function when teeth were lost after segmental mandibulectomy and bone reconstruction. The results of our study are concordant with the report. The overall efficiency of mastication was usually performed the patients' questionnaire $(18,42)$. Wax et al reported on 17 patients who underwent condyle reconstruction with a fibula free graft and found that four patients needed a liquid diet and four patients remained on a nasogastric (NG) feeding tube (3). Although our sample size was small, there were no patients remained on a liquid diet or were NG-tube dependent. Thus, our patients exhibited 
almost good food tolerance and masticatory function after condylar reconstruction.

In this study, we evaluated the morphological and functional outcome following the stereolithographic model-assisted reconstruction of the condyle with placement of a vascularized free fibula into the glenoid fossa. The stereolithography model could accurately reproduce the 3D shape and anatomical location of the original condyle with the aid of MMF. MMF could also support in the determination of a proper position for the bone flap to ensure the future dental rehabilitation. The correct occlusion and optimal relationship between the condyle and the glenoid fossa were achieved. The end of the fibular bone graft recapitulated the native condylar anatomy and function, and served morphologically and functionally as a neocondyle. The reservation of the disc and molding of the end of the fibular graft seemed to be responsible for the good outcome in terms of function. All the patients showed good food tolerance and masticatory function after reconstruction.

Although the type of condylar reconstruction remains controversial, the stereolithographic model-assisted reconstruction of the mandibular condyle with a vascularized fibular flap is useful for reconstructions of the hemimandible, including condylar defects.

\section{References}

1. Potter JK and Dierks EJ: Vascularized options for reconstruction of the mandibular condyle. Semin Plast Surg 22: 156-160, 2008.

2. Iñigo F, Rojo P, Ysunza A and Jimenez Y: Three different techniques for mandibular reconstruction after hemimandibulectomy. J Craniofac Surg 8: 58-64, 1997.

3. Wax MK, Winslow CP, Hansen J, MacKenzie D, Cohen J, Andersen P and Albert T: A retrospective analysis of temporomandibular joint reconstruction with free fibula microvascular flap. Laryngoscope 110: 977-981, 2000.

4. Guyot L, Richard O, Layoun W, Cheynet F, Bellot-Samson V, Chossegros C, Blanc JL and Gola R: Long-term radiological findings following reconstruction of the condyle with fibular free flaps. J Craniomaxillofac Surg 32: 98-102, 2004.

5. Engroff SL: Fibula flap reconstruction of the condyle in disarticulation resections of the mandible: A case report and review of the technique. Oral Surg Oral Med Oral Pathol Oral Radiol Endod 100: 661-665, 2005

6. Khariwala SS, Chan J, Blackwell KE and Alam DS: Temporomandibular joint reconstruction using a vascularized bone graft with Alloderm. J Reconstr Microsurg 23: 25-30, 2007.

7. González-García R, Naval-Gías L, Rodríguez-Campo FJ, Martínez-Chacón JL and Gil-Díez Usandizaga JL: Vascularized fibular flap for reconstruction of the condyle after mandibular ablation. J Oral Maxillofac Surg 66: 1133-1137, 2008.

8. Thor A, Rojas RA and Hirsch JM: Functional reconstruction of the temporomandibular joint with a free fibular microvascular flap. Scand J Plast Reconstr Surg Hand Surg 42: 233-240, 2008.

9. Moore EJ and Hamilton SS: Mandibular condyle reconstruction with fibula free-tissue transfer. Ear Nose Throat J 91: E18-E24, 2012

10. Wang WH, Zhu J, Deng JY, Xia B and Xu B: Three-dimensional virtual technology in reconstruction of mandibular defect including condyle using double-barrel vascularized fibula flap. J Craniomaxillofac Surg 41: 417-422, 2013.

11. Bredell M, Grätz K, Obwegeser J and Gujer AK: Management of the temporomandibular joint after ablative surgery. Craniomaxillofac Trauma Reconstr 7: 271-279, 2014.

12. Chao JW, Rohde CH, Chang MM, Kutler DI, Friedman J and Spector JA: Oral rehabilitation outcomes after free fibula reconstruction of the mandible without condylar restoration. J Craniofac Surg 25: 415-417, 2014.

13. Hoang MP, Nguyen TT, Nguyen LK and Jeng SF: Ossifying fibroma of the mandible: A case report using vascularized free fibula flap reconstruction. Plast Reconstr Surg Glob Open 3: e470,2015.
14. Smith M, Williams F and Ward BB: Hard tissue reconstruction. In: Management of Complications in Oral and Maxillofacial Surgery. Miloro M and Kolokythas A (eds). 1st edition. Wiley-Blackwell, Oxford, pp283-316, 2012.

15. Antony AK, Chen WF, Kolokythas A, Weimer KA and Cohen MN: Use of virtual surgery and stereolithography-guided osteotomy for mandibular reconstruction with the free fibula. Plast Reconstr Surg 128: 1080-1084, 2011.

16. List MA, Ritter-Sterr C and Lansky SB: A performance status scale for head and neck cancer patients. Cancer 66: 564-569, 1990.

17. Urken ML, Weinberg H, Vickery C, Buchbinder D, Lawson W and Biller HF: Oromandibular reconstruction using microvascular composite free flaps. Report of 71 cases and a new classification scheme for bony, soft-tissue, and neurologic defects. Arch Otolaryngol Head Neck Surg 117: 733-744, 1991.

18. Curtis DA, Plesh O, Miller AJ, Curtis TA, Sharma A, Schweitzer R, Hilsinger RL, Schour L and Singer M: A comparison of masticatory function in patients with or without reconstruction of the mandible. Head Neck 19: 287-296, 1997.

19. Vega LG, González-García R and Louis PJ: Reconstruction of acquired temporomandibular joint defects. Oral Maxillofacial Surg Clin North Am 25: 251-269, 2013.

20. Petruzzelli GJ, Cunningham K and Vandevender D: Impact of mandibular condyle preservation on patterns of failure in head and neck cancer. Otolaryngol Head Neck Surg 137: 717-721, 2007.

21. Marx RE, Cillo JE Jr, Broumand V and Ulloa JJ: Outcome analysis of mandibular condylar replacements in tumor and trauma reconstruction: A prospective analysis of 131 cases with long-term follow-up. J Oral Maxillofac Surg 66: 2515-2523, 2008.

22. Carlson ER: Disarticulation resections of the mandible: A prospective review of 16 cases. J Oral Maxillofac Surg 60: 176-181, 2002.

23. Rosenberg AJ, Van Cann EM, van der Bilt A, Koole R and van Es RJ: A prospective study on prognostic factors for free-flap reconstructions of head and neck defects. Int J Oral Maxillofac Surg 38: 666-670, 2009.

24. Nahabedian MY, Tufaro A and Manson PN: Improved mandible function after hemimandibulectomy, condylar head preservation, and vascularized fibular reconstruction. Ann Plast Surg 46: 506-510, 2001.

25. Xu LQ, Zhang CP, Poh EH, Yin XL and Shen SK: A novel fibula osteotomy guide for mandibular reconstruction. Plast Reconstr Surg 129: 861e-863e, 2012.

26. Hanasono MM and Skoracki RJ: Computer-assisted design and rapid prototype modeling in microvascular mandible reconstruction. Laryngoscope 123: 597-604, 2013.

27. Lim SH, Kim MK and Kang SH: Precision of fibula positioning guide in mandibular reconstruction with a fibula graft. Head Face Med 12: 7, 2016.

28. Mast G: Techniques of mandibulomaxillary fixation (MMF) In: Principles of Internal Fixation of the Craniomaxillofacial Skeleton. Ehrenfeld M, Manson PN and Prein J (eds). 1st edition. George Thieme Verlag, New York, NY, pp115-123, 2012.

29. Markowitz BL, Roumanas E and Calcaterra T: Surgical stents for composite mandible reconstruction. Plast Reconstr Surg 96: 194-200, 1995

30. Yoshimura H, Ohba S, Yasuta M, Nakai K, Fujieda S and Sano K: Infrazygomatico-coronoid fixation in a segmental mandibular reconstruction with a free vascularized flap: A simple and correct repositioning method without interfering with reconstructive and microsurgical procedures. Head Neck 38: 1679-1687, 2016.

31. McDonald F: The condylar disk as a controlling factor in the form of the condylar head. J Craniomandib Disord 3: 83-86, 1989.

32. Hamada Y, Kondoh T, Takada N and Seto K: MRI and arthroscopic findings in the temporomandibular joint after mandibulectomy including the unilateral condyle. Report of two cases. Int J Oral Maxillofac Surg 29: 341-343, 2000.

33. Miyamoto H, Kurita K, Ishimaru J and Goss AN: A sheep model for temporomandibular joint ankylosis. J Oral Maxillofac Surg 57: 812-817, 1999.

34. Porto G, Vasconcelos B and Silva V Jr: Development of temporomandibular joint ankylosis in rats: A preliminary experimental study. Int J Oral Maxillofac Surg 37: 282-286, 2008

35. Dai J, Ouyang N, Zhu X, Huang L and Shen G: Injured condylar cartilage leads to traumatic temporomandibular joint ankylosis. J Craniomaxillofac Surg 44: 294-300, 2016. 
36. Xiang GL, Long X, Deng MH, Han QC, Meng QG and Li B: A retrospective study of temporomandibular joint ankylosis secondary to surgical treatment of mandibular condylar fractures. Br J Oral Maxillofac Surg 52: 270-274, 2014.

37. Beumer J III, Marunick MT, Silverman S Jr, Garrett N, Rieger J, Abemayor E, Penn R, Nabili V, Rezaee R, Curtis DA, et al: Rehabilitation of tongue and mandibular defects. In: Maxillofacial Rehabilitation: Prosthodontic and Surgical Management of Cancer-Related, Acquired and Congenital Defects of the Head and Neck. Beumer J III, Marunick MT and Salvatore JE (eds). 3rd edition. Quintessence Publishing, Hanover Park, pp61-154, 2011.

38. Brand RW and Isselhard DE: Temporomandibular joint. In: Anatomy of Orofacial Structures. 7th edition. Mosby, St. Louis, pp348-352, 2003.

39. Crawford SD: Condylar axis position, as determined by the occlusion and measured by the CPI instrument, and signs and symptoms of temporomandibular dysfunction. Angle Orthod 69: 103-116, 1999 .
40. Linsen S, Schmidt-Beer U, Fimmers R, Grüner M and Koeck B: Craniomandibular pain, bite force, and oral health-related quality of life in patients with jaw resection. J Pain Symptom Manage 37: 94-106, 2009

41. Marunick MT and Mathog RH: Mastication in patients treated for head and neck cancer: A pilot study. J Prosthet Dent 63: 566-573, 1990.

42. Boretti G, Bickel M and Geering AH: A review of masticatory ability and efficiency. J Prosthet Dent 74: 400-403, 1995.

43. Tan ZZ, Liu B, Wei JX, Zou H and Zhao YF: Effects of mandibular odontogenic keratocyst surgery and removable partial prostheses on masticatory performance. J Prosthet Dent 97: 107-111, 2007. 